\title{
KintSUGi IMAGING OF BATTERY ELECTRODES: Distinguishing PORES FROM THE CARBON BINDER DOMAIN USING PT DEPOSITION
}

PREPRINT - JULY 10, 2022

\author{
Samuel J. Cooper ${ }^{* 1}$, Scott A. Roberts ${ }^{2}$, Zhao Liu ${ }^{3}$, and Bartłomiej Winiarski ${ }^{4}$ \\ ${ }^{1}$ Dyson School of Design Engineering, Faculty of Engineering, Imperial College London, U.K. \\ ${ }^{2}$ Thermal/Fluid Component Sciences Department, Engineering Sciences Center, \\ Sandia National Laboratories, Albuquerque, NM, U.S.A. \\ ${ }^{3}$ Thermo Fisher Scientific, 5350 NE Dawson Creek Dr, Hillsboro, OR, U.S.A. \\ ${ }^{4}$ Thermo Fisher Scientific, Pecha 1282/12, 62700 Brno, Czech Republic
}

\begin{abstract}
The mesostructure of porous electrodes used in lithium-ion batteries strongly influences cell performance. Accurate imaging of the distribution of phases in these electrodes would allow this relationship to be better understood through simulation. However, imaging the nanoscale features in these components is challenging. While scanning electron microscopy is able to achieve the required resolution, it has well established difficulties imaging porous media. This is because the flat imaging planes prepared using focused ion beam milling will intersect with the pores, which makes the images hard to interpret as the inside walls of the pores are observed. It is common to infiltrate porous media with resin prior to imaging to help resolve this issue, but both the nanoscale porosity and the chemical similarity of the resins to the battery materials undermine the utility of this approach for most electrodes.

In this study, a technique is demonstrated which uses in situ infiltration of platinum to fill the pores and thus enhance their contrast during imaging. Reminiscent of the Japanese art of repairing cracked ceramics with precious metals, this technique is referred to as the kintsugi method. The images resulting from applying this technique to a conventional porous cathode are presented and then segmented using a multi-channel convolutional method. We show that while some cracks in active material particles were empty, others appear to be filled (perhaps with the carbon binder phase), which will have implications for the rate performance of the cell. Energy dispersive X-ray spectroscopy was used to validate the distribution of phases resulting from image analysis, which also suggested a graded distribution of the binder relative to the carbon additive. The equipment required to use the kintsugi method is commonly available in major research facilities and so we hope that this method will be rapidly adopted to improve the imaging of electrode materials and porous media in general.
\end{abstract}

\section{Introduction}

The performance of lithium-ion batteries is closely linked to the mesostructure of their porous electrodes. These electrodes must simultaneously enable transport of lithium ions through their electrolyte-filled pores, conduction of electrons through the solid phases, diffusion of lithium between the surface and core of the active materials, and exchange of lithium and electrons at the interface between the active material and the electrolyte. These structures must also be sufficiently mechanically robust to withstand the morphological distortions caused by changes in lithiation state, temperature, and external pressure loads. In order to design better electrodes, it is critical to understand the relationship between morphology and performance, which in turn requires observing the mesostructure accurately. Detailed images that resolve the mesostructure can provide direct insight into performance through visual inspection, but can also feed detailed image-based simulations of electrode performance $[1,2]$.

A conventional lithium-ion battery cathode might contain roughly spherical particles of an active material (AM), such as lithium nickel manganese cobalt oxide (NMC), held together by a conductive binder, known as the Carbon/Binder Domain (CBD). CBD is typically a mixture of a carbon black, used to enhance the electronic conductivity of the solid phase, and a polymeric binder (e.g. polyvinylidene fluoride), used to hold the AM and carbon together. While the electrode is primarily composed of the AM phase, with AM typically making up 90-96 weight percent, the less dense

\footnotetext{
${ }^{*}$ Corresponding author: samuel.cooper@imperial.ac.uk
} 
CBD can account for 20-30 percent of the electrode solid volume and its morphology strongly influences both the electronic transport properties of the solid and the ionic transport through the pores $[3,4]$.

X-ray Computed Tomography (XCT) and Focused Ion Beam Scanning Electron Microscopy (FIB-SEM) have both been used to image battery electrodes at the microscale $[5,6,7,8]$. The past decade has seen improvements in acquisition time (more sensitive detectors) [9], resolution [10,11], and phase sensitivity (phase contrast XCT $[12,13]$ and electron energy-selective detection [14]). However, several key challenges remain when trying to capture all relevant features using either approach [15].

The principal limitation of XCT is its inability to convincingly observe the CBD. Firstly, the geometrical features of the CBD are at the limits of the resolution capability of XCT $(\sim 50 \mathrm{~nm})$, even given powerful synchrotron X-ray sources (Fig. 1) [13,16]. Secondly, NMC, like most cathode active materials, attenuates $\mathrm{X}$-rays much more strongly than the CBD. This means that, once the X-ray projection images have been obtained and reconstructed, only the particles of active material are clearly visible, as the CBD is typically indistinguishable from noise in the pore phase [6]. Attempts to segment CBD from AM phases in these images is fraught with speculation and the results are far from convincing $[17,18]$.

Phase contrast synchrotron XCT has been used to try and resolve this $[12,13]$, but the results are still not at the resolution $(<30 \mathrm{~nm})$ achievable by electron microscopy based 3D serial sectioning tomography. Furthermore, access to synchrotron imaging lines is limited compared to many lab based XCT and FIB-SEM systems. Others have added iron nanoparticles into the CBD during manufacturing in order to increase its contrast [19]. As a result of this difficulty, it is not uncommon to resort to inserting the CBD into the microstructure images using statistical methods after segmentation of the active material $[20,21]$.

The FIB-SEM "slice and view" serial sectioning method is typically able to image at higher resolutions than XCT ( $\geq 1 \mathrm{~nm}$ image resolution, $\geq 5 \mathrm{~nm}$ slice thickness) [22]. Although for Ga liquid metal ion source FIBs [23] this high resolution has generally also meant a small cross-sectional field of view; the advent of Plasma FIB (PFIB) columns and femtosecond lasers has extended the usable field of view to hundreds of microns (PFIB)[24] or even millimeters (laser) [25]. As opposed to FIB-SEM, XCT is often referred to as a "non destructive" imaging technique. However, the sample preparation required to use XCT at these high resolutions (sometimes even including PFIB or laser ablation) effectively makes it destructive in many cases, as although the imaged region is preserved, any surrounding material would have been removed.

When a set of 2D FIB-SEM images are stacked to create a $3 \mathrm{D}$ volume, it is necessary that the information in each image is from a flat imaging plane in the sample. However, if the material is porous, the SEM simply images the back walls of any pores that intersect the imaging plane, which results in information from different planes of the sample being mixed together. In these situations, SEM's large focal depth becomes a disadvantage. For rigid materials with percolated pore networks, this issue is conventionally overcome by infiltrating the pores with epoxy resin before imaging. Once the resin has cured, the sample is fully dense and any signal from the resin is simply interpreted as belonging to the pore phase following reconstruction. This has been used to great effect in cermet fuel cell electrodes [26]. However, in the case of lithium-ion battery electrodes, e.g. in Fig. 1, this is problematic for three distinct reasons. Firstly, and most importantly, any carbon based resin will have a very similar signal to the CBD phase, making it difficult to distinguish between CBD and resin. Secondly, resins tend to be fairly viscous and the pores of the CBD phase are very small, so complete infiltration is challenging (closed pores are also inaccessible). Lastly, many battery electrodes are not entirely rigid and so any shrinkage of the epoxy while curing will distort the mesostructure.

Several approaches have been suggested for resolving some of these issues. To improve the contrast between the carbon phase and the epoxy Ghosh et al. [27] infiltrated a carbon paper with a cobalt doped epoxy. Biton et al. [28] doped their epoxy with bromine and Ender et al. [29] used silicone resin to distinguish carbon from pore in an LFP electrode. Lagadec et al. [30, 31] doped butter with osmium as a replacement for epoxy as infiltrant. Vierrath et al. [32] effectively infiltrated the nanopores of the CBD through atomic layer deposition of $\mathrm{ZnO}$ before the sample was imaged. This approach showed excellent contrast, although any isolated pore volume was not infiltrated.

Rather than infiltration, Moroni and Thiele [33] developed an "optical flow" approach to analyze the images containing pore inner surface information. ArgandaCarreras et al. [34] used a machine learning (ML) based advanced segmentation approach to learn the distinction between in plane and pore back information. However, even these powerful new digital techniques are only as good as the quality of their training data [13] and if the operator cannot distinguish the pore from CBD then this error will propagate throughout [18]. Well defined reference models are very effective in rapid segmentation of hundreds of similar mesostructures, with the time developing the reference model being paid off through many inferences. However, when only a few volumes are available, the cost of developing reference ML models and associated data processing will not necessarily save time over classical segmentation.

Recently, [35] implemented convolutional neural networks (CNNs) to segment some cathode samples; however, in order to train the models on such scarce data (whilst avoiding overfitting), it was necessary to include artificial data generated from simulated par- 


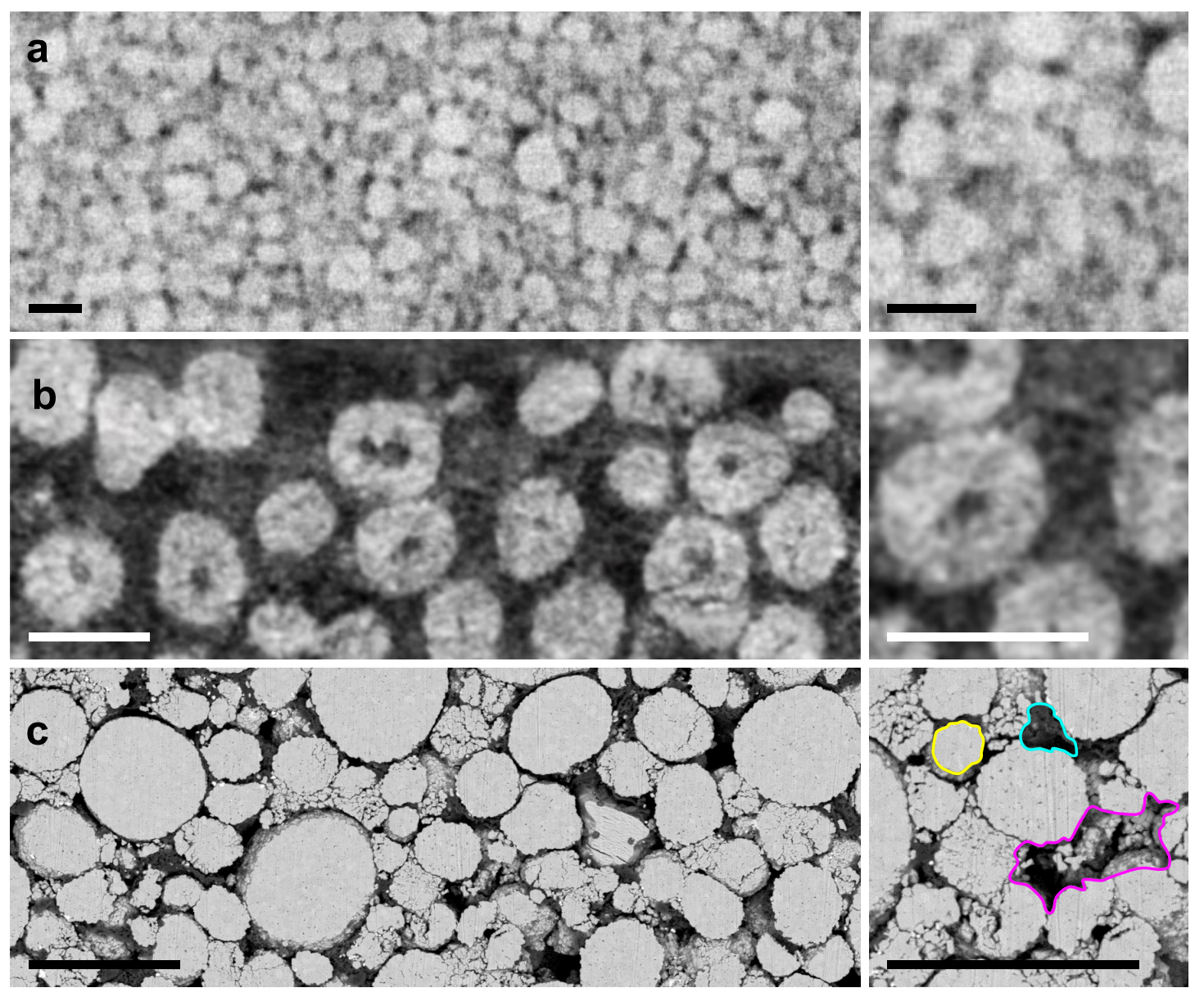

Figure 1: NMC cathodes imaged using different techniques. Right column is a closer view of the central region of the left column. (a) Typical virtual cross-section of the cathode reconstructed from series of X-Ray attenuation projections collected via a commercial micro CT (400 nm pixels). (b) Typical virtual cross-section of the cathode reconstructed from series of X-Ray phase contrast projections collected with a synchrotron hard beam at ESRF ID16A-NI beamline (50 nm pixels) [13]. (c) Typical cross-section of NMC cathode prepared with Xe Plasma FIB-SEM instrument and imaged with SEM-BSE detector $(20 \mathrm{~nm}$ pixels). The region highlighted in yellow shows cross sectioned particle on the imaging plane. The region highlighted in cyan shows what is probably a cross sectioned patch of CBD on the imaging plane. The region highlighted in magenta has ambiguous content as it appears to be the inner surface of an uninfiltrated pore. All scale bars are $20 \mu \mathrm{m}$.

ticle arrangements combined with various expected artefacts. Although some satisfying looking segmentations are generated, the approach requires the user to know the shapes that they are expecting to see in advance, in order to generate the simulated structures. This might be appropriate in scenarios where the same type of material is being repeatedly imaged, although even then the segmentation algorithm may become effectively blind to any features that it was not expecting to see and should therefore be used with caution.

In this study, we describe a method for the imaging of porous battery electrode mesostructures with excellent phase identifiability, as well as a multichannel convolutional approach for segmenting the resulting data. The imaging method does not require preinfiltration with resin or inference methods like optical flow. Instead, once an imaging plane has been exposed by PFIB milling, the pores are filled in by depositing platinum, as a kind of in situ infiltration. This is achieved by first depositing a thick layer of platinum over the whole surface (c. $1 \mu \mathrm{g}$ for this image, which would add minimal cost, although other metals such as Tungsten may be used) and then carefully ion-milling it away until the imaging plane has returned to the location it was before the platinum was deposited. This leaves platinum only in the pores (even those that do not globally percolate), which are now easy to distinguish from the CBD and AM, leading to accurate segmentation. Eswara-Moorthy et al. [36] also used in situ Pt infiltration to image porous carbon and showed it to be very effective. This method can be applied to study any micro-porous materials, although battery cathodes are an ideal case study due to their multi-phase and multi-length-scale nature. The work presented here is closely related to the author's previous work on sup- 
pressing curtaining effects during serial sectioning in additively manufactured metals containing gas pores and large open cracks [37].

\section{Methods}

The imaging method is a form of iterative, localised, in situ infiltration where, once an imaging plane has been prepared with the PFIB, a thick layer of platinum is deposited onto its surface, thus also infiltrated into any open pores. Next, the PFIB is used to gently polish away any excess platinum (i.e. that not inside a pore or crack). The end result is a flatter imaging plane, where the regions that were previously open pores are now easy to distinguish due to their distinct platinum signal. This method is reminiscent of the traditional Japanese art of repairing broken pottery using precious metals known as kintsugi ("golden joinery") and is referred to as the kintsugi method throughout this paper. Since platinum was used rather than gold, the name hakkinntsugi might be more literally correct; however, deposited gold would work equally well and kintsugi is the better known and more evocative term. Kintsugi also has a poetic meaning, where it alludes to the deliberate emphasising of imperfections, which is also particularly apt here.

\subsection{Electrode material}

The sample we used to demonstrate this approach is a lithium nickel manganese cobalt oxide (NMC) cathode disassembled from a fresh 18650 Li-ion battery cell, rinsed, and dried to remove the liquid electrolyte. The cathodes are around 80 microns thick and are coated on either side of a $c$. 20 microns thick aluminium current collector foil. A $5 \times 15 \mathrm{~mm}^{2}$ sample is mounted using an Agar fast drying silver suspension (AGG3691) on a $45^{\circ}$ pre-tilted scanning electron microscope stub (Fig. 2a). In so doing, the edge of the specimen is exposed for cross-section preparation and top-down SEM imaging with back-scattered electron retractable detector.

\subsection{Cross-sectioning}

The sample on a stub is mounted in a vacuum chamber of a Thermo Scientific Helios ${ }^{\mathrm{TM}} \mathrm{Hydra}^{\mathrm{TM}}$ Plasma Focused Ion Beam - Scanning Electron Microscope (PFIB-SEM). We used Xenon ions (Hydra PFIB-SEM is equipped with four ion species: $\mathrm{Xe}, \mathrm{Ar}, \mathrm{O}$ and $\mathrm{N}$.) for the site preparation, cross-sectioning, and contrast enhancing amorphous composite layer deposition from carbon $(\mathrm{C})$ and platinum $(\mathrm{Pt})$ gaseous precursors.

The site for cross-sectioning is prepared in the typical way used for serial sectioning tomography. First, a narrow protective layer $(200 \mu \mathrm{m} \times 30 \mu \mathrm{m} \times 5$ $\mu \mathrm{m})$ composite made of $\mathrm{Pt}$ and $\mathrm{C} \mathrm{mix}^{2}$ is deposited from MultiChem ${ }^{\mathrm{TM}}$ gaseous precursors delivery system using $25 \%$ of maximum plasma acceleration volt-

\footnotetext{
${ }^{2} 100 \%$ and $2 \%$ duty cycle for Pt and C, respectively
}

age (Fig. 2a). A block face of about $200 \mu \mathrm{m}$ wide $\times 230 \mu \mathrm{m}$ deep is shaped using $30 \mathrm{kV}$ and $2500 \mathrm{nA}$ beam, where two trenches are milled on both sides of the block face. This procedure rapidly creates a sharply defined block face (Fig. 2b). Next, a fiducial " $x$ "-shaped marker is milled on the protective layer using $30 \mathrm{kV} 15 \mathrm{nA}$ beam. The fiducial is used for precise positioning of the focused beam during automated polishing run of the cross-section with Auto Slice \& View (ASV) software. The automated polishing is done with PFIB at $30 \mathrm{kV}$ and $60 \mathrm{nA}$. We used the rocking polish method with $3^{\circ}$ beam tilts and $25 \mathrm{~nm}$ cutting beam advancements for each rocking polish position to generate curtain-free cross-sections [24].

\subsection{Kintsugi method}

The new cross-sectional surface contrast enhancement method for back-scattered electron SEM imaging consists of iteratively depositing and then removing platinum before imaging (Fig. $2 \mathrm{~d}$ and e). The final surface for SEM imaging is achieved after several repetitions of this process. The number of iterations depends on the porosity level and size of voids. For example, surfaces with shallow recesses (depth $<20 \%$ of the width) are ready for imaging after a few rounds. However, as discussed later, it was found not to be necessary to fill the deepest pores. For the battery cathode sample presented in this study, we deposited a thick Pt layer (c. $2 \mu \mathrm{m}$ ) on the block face (Fig. 2c). Next, about 1.5 microns of material is removed. This is known as "green clean" step in the ASV run (Fig. 2d). These settings allow defining flat and smooth block face with minimal evidence of the curtaining effect. Finally, a regular automated ASV run is set with the cutting parameters presented in the previous section.

\subsection{Imaging}

In the ASV run we defined multi-detector SEM (the Elstar electron column) imaging conditions optimised for capturing a vivid signal from the top most superficial plane region of constituent material of the cathode. In so doing we used a $5 \mathrm{kV}$ and $1.6 \mathrm{nA}$ electron beam and a $4 \mathrm{~mm}$ working distance. We collected simultaneously the back scattered electrons (BSE) using: (1) the retractable annular "concentric back-scattered" (CBS) electron detector with two inner rings ( $\mathrm{A}$ and $\mathrm{B}$ ) active; and (2) the through-lens detector (TLD), which captures the few electrons scattered directly back towards the beam. The electron beam scanned the specimen surface with $3 \mu$ s dwell time collecting $4096 \times 3816$ pixels images, where 1 pixel is about $20 \mathrm{~nm}$. The SEM image brightness and contrast levels were adjusted to optimise the visibility of the AM and CBD. During the ASV run, SEM images were captured every 4th PFIB cut, thus after removing $100 \mathrm{~nm}$ layer of material.

Since the CBS detector sits between the SEM polepiece and right above the sample, the number of BSE 


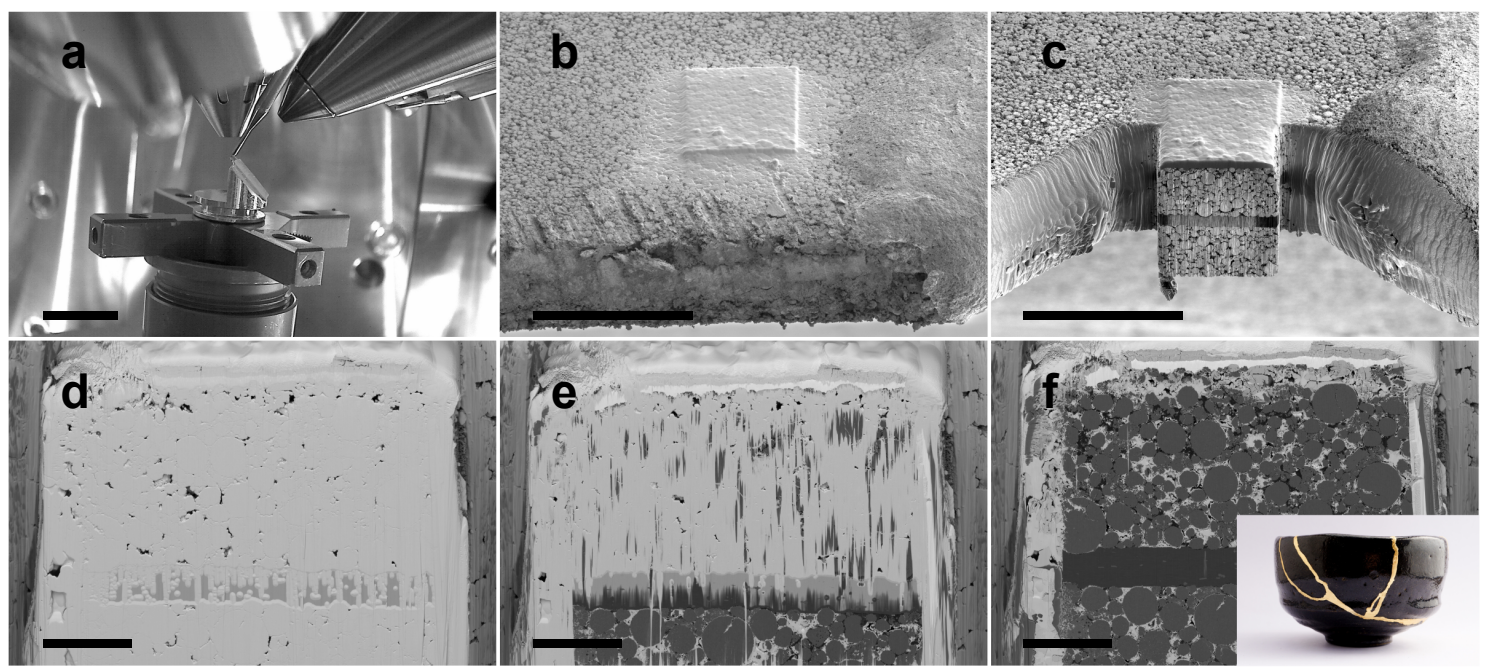

Figure 2: The cross-section preparation workflow using Helios Hydra PFIB-SEM. (a) Image of the sample arrangement relative inside the vacuum chamber. (b) deposition of the $\mathrm{Pt}-\mathrm{C}$ mix protective layer on the top surface of cathode. (c) the material block and block face preparation. (d) Pt layer deposition on the block face; (e) the automated ASV run, removing the excess of Pt layer. (f) image of the kintsugi infiltrated block face at $54 \mathrm{~nm}$ resolution (Inset shows an example of the pot repaired using the traditional kintsugi method). The scale bar in (a) is $8 \mathrm{~mm}$; in (b, c) is $200 \mu \mathrm{m}$; and in (d-f) is $40 \mu \mathrm{m}$.

electrons detected greatly exceeds the signal-to-noise level (SNL), and as a result the images are very sharp. Although the TLD has a far lower signal, and is therefore noisier, it is much less sensitive to topology and captures electrons both from the top surfaces and those coming from the pore-backs. Although in general the image from the CBS detector is superior, the additional information from the TLD is complementary and therefore helpful for segmentation, as explained in the following section.

The chemical composition of the freshly exposed cross-section we determined in situ using energy dispersive X-ray spectroscopy (EDS). The Elstar electron column accelerated electrons from the extractor to $10 \mathrm{keV}$ landing energy. The $1.6 \mathrm{nA}$ electron beam rastered (54 nm step size) the surface area of $72.8 \mu \mathrm{m}$ $\times 52 \mu \mathrm{m}$ positioned horizontally at $4.10 \mathrm{~mm}$ working distance from the column pole-piece. The EDS map $(1358 \times 970$ pixels $)$ was collected and processed using Oxford Instruments X-Max 50 silicon drift detector and Aztec 5.0 analytical suite. We used the following EDS map parameters in Aztec: energy range $10 \mathrm{keV}$; 2048 channels; 5 eV/channel; 10 completed frames; process time ${ }^{3}$ 3; live time 1317 s; 3,425,962 total counts in TruMap ${ }^{4}$ signal processing engine.

\subsection{Segmentation}

The data was segmented using the open-source Trainable Weka Segmentation (TWS) tool [34], which is a plugin available as part of the Fiji [38] distribution of
ImageJ [39]. TWS is a convolution-based image segmentation tool (2D or 3D), in which a classifier is iteratively trained based on user-generated labels. The supervised machine learning models in TWS are fast and easy to use; however, many of the options available to the user (such as convolutional kernel type or classifier algorithm) must be selected somewhat arbitrarily. Five classes were defined during training to allow the classifier to group similar regions more easily: (1) current collector, (2) active material, (3) binder, (4) platinum at the pore edge, and (5) platinum at depth.

As discussed in the following section, although it is in principle possible to completely fill pores of any depth with deposited platinum, this was found to be both time consuming and unnecessary. This is because phase (5) is always surrounded by a ring of phase (4), making it is easy to distinguish from the binder. Once segmentation was complete, classes (4) and (5) were simply merged as they both can be attributed to the pore phase.

Although it was possible to achieve an excellent segmentation with the SEM-BSE image alone, it was found that an even better segmentation could be generated (with fewer labels) using the information from the TLD image as well. In order to enable this, the two images were concatenated into a stack and then loaded into TWS in its 3D segmentation mode. This is not the intended use of this mode, but it is an effective way to make use of multimodal image data. Crucially, only the segmentation of the BSE layer is eventually saved and that of the TLD image layer is discarded.

\footnotetext{
${ }^{3}$ The Process Time $\left(\begin{array}{ll}1 \ldots & 6\end{array}\right)$ is the length of time spent reducing noise from the X-ray signal coming from the EDS detector during processing.

${ }^{4}$ TruMap deconvolutes overlapping peaks and removes false variations due to $\mathrm{X}$-ray background.
} 

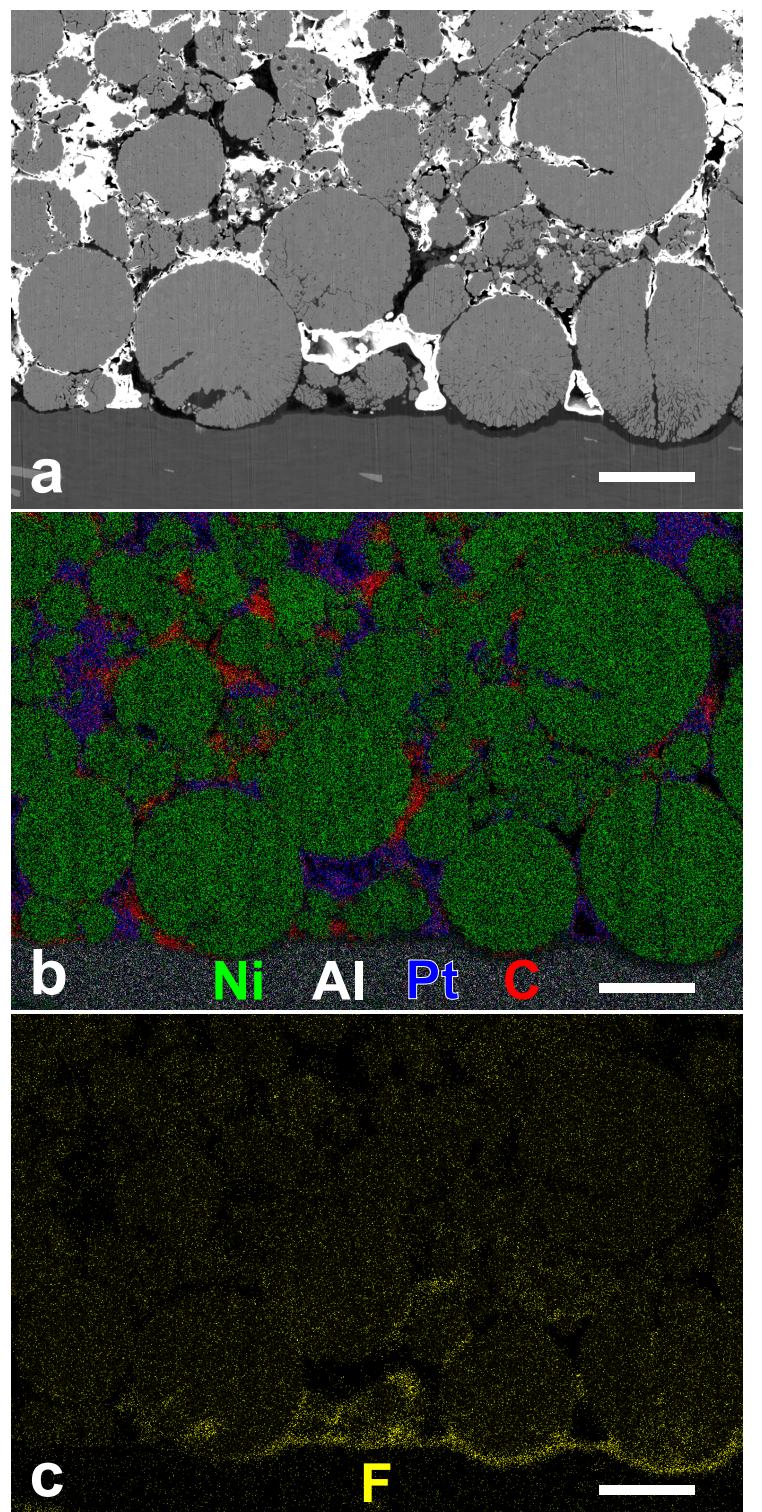

Figure 3: Kintsugi method results. (a) SEM-BSE cross-section image using CBS detector. (b) Composite of EDS images highlighting the distributions of $\mathrm{Al}$ (current collector), Ni (AM particles), Pt (pores), and $\mathrm{C}$ (CBD). (c) EDS image highlighting the presence of $\mathrm{F}$ (probably from the polyvinylidene fluoride binder). All scale bars at $10 \mu \mathrm{m}$.

It should be noted that no filters were applied before segmentation using TWS and the authors strongly recommend this as best practice to others performing image analysis. This is because filtering can only remove information from images and any perceived benefit created by "removing noise" (e.g. using Gaussian blur) can be learned in the filters of a convolution method.

\section{Results and Discussion}

We demonstrate in Fig. 3 that the kintsugi method generates excellent contrast between all of the relevant phases in a single cross-section of the electrode. This was further validated with EDS data that provided confirmation that the easily distinguished greyscale phases belong to their expected material. Unsurprisingly, visual inspection clearly shows large, mostly spherical NMC particles atop an Al current collector, where the $\mathrm{Ni}$ and $\mathrm{Al}$ EDS signals uniquely identify the particle and collector phases, respectively (Fig. 3).

For the first time, we can also confidently distinguish between the pore and CBD phases in the BSE image. Because the pores are mostly filled with $\mathrm{Pt}$, they show up bright white in the BSE Fig. 3, which is confirmed through the Pt EDS signal in Fig. 3. Even though the pores are not entirely filled with $\mathrm{Pt}$, the multichannel convolutional segmentation approach is easily trained to distinguish pores from CBD. The remaining phases, which are a darker grey in the BSE image, are CBD, which is uniquely identified by the combination of the F (Fig. 3) and C (Fig. 3) EDS signals. Due to the kintsugi method, there is no longer any ambiguity between the CBD and the back walls of pores (which would still show up as C or F EDS signals).

Additional microstructural details become more clearly visible in a zoomed subdomain of the BSE image shown in Fig. 4b. The first is that there is heterogeneity within the cracks in the NMC particles. Because these NMC particles are actually secondary particles composed of nanoscale primary particles, cracks within the secondary particles are commonplace. However, there is no definitive view in the literature as to whether these cracks are filled with CBD or remain empty to later fill with electrolyte. This uncertainty will result in errors in predicting performance. Cracks that remain empty may act as pathways to enhance transport in the system, whereas CBD-filled cracks will block this effect. By observing Fig. 4b, we see that the answer is "both." On the left side of the large particle at the bottom left of the image towards the current collector, the large horizontal crack, along with the smaller cracks, are all uniformly and completely filled with CBD. However, on the right side of the same particle, the larger crack is partially filled with CBD deeper in the particle, but towards the surface the crack is empty (i.e. filled with Pt during imaging). Note that because we only have a single imaging plane, we do not know whether this open region is towards the middle or at the edge of the crack or particle. Very possibly this plane is towards the edge, where the crack could have additionally opened up later in the manufacturing process, possibly during the calendaring process. Regardless, the kintsugi approach clearly shows the capability to differentiate both cases, with $\mathrm{CBD}$ and pore in NMC particle cracks. 

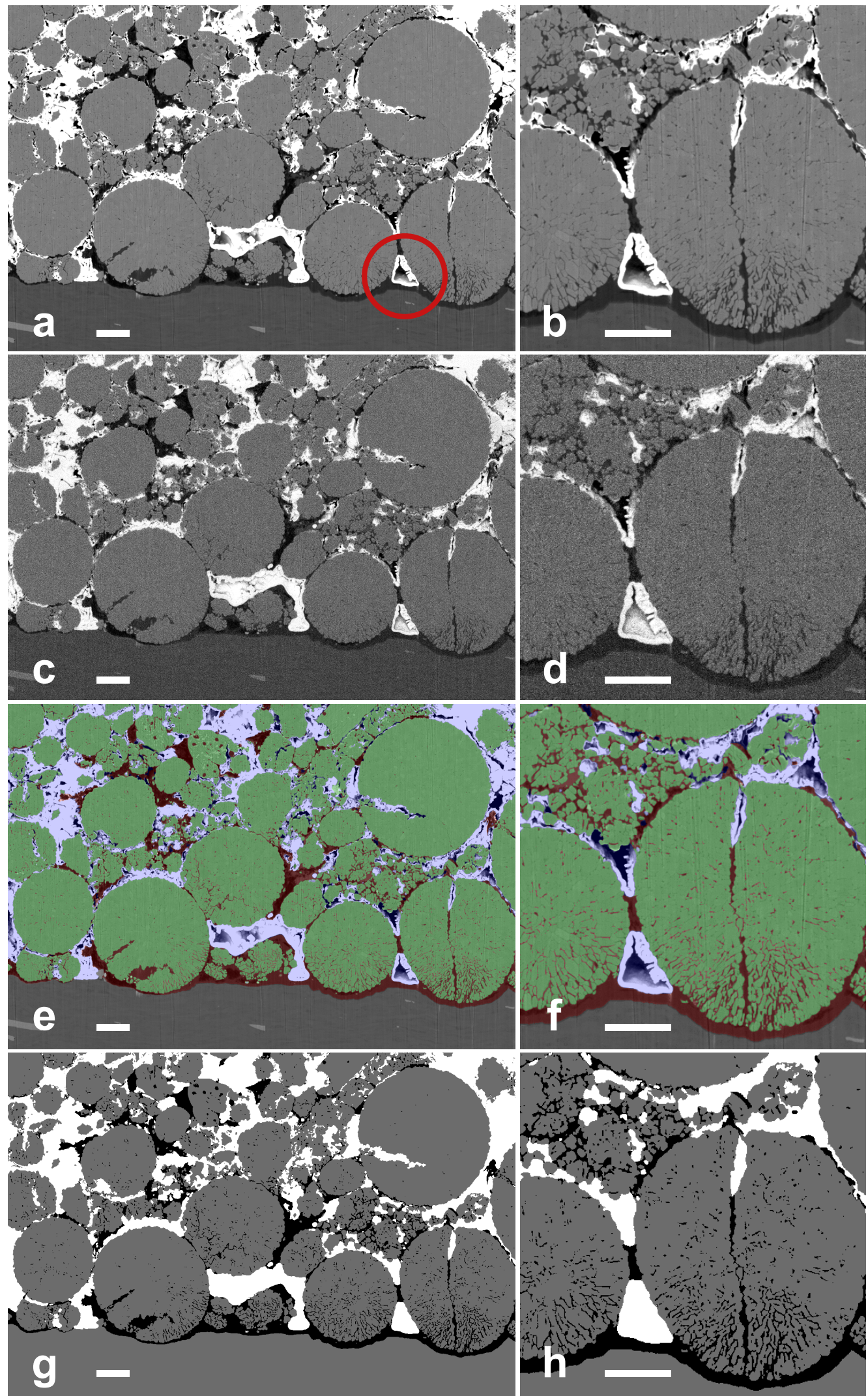

Figure 4: Images of cathode cross-section prepared using kintsugi method. (a-b) SEM-BSE images using CBS detector. (c-d) SEM-BSE images using TLD. (e-f) CBS image overlaid with segmentation false colouring, where green is active material, red is CBD, and blue is pore. (g-h). Segmentation where white is pore, black is binder and grey is either active material or current collector. All scale bars are $5 \mu \mathrm{m}$. 


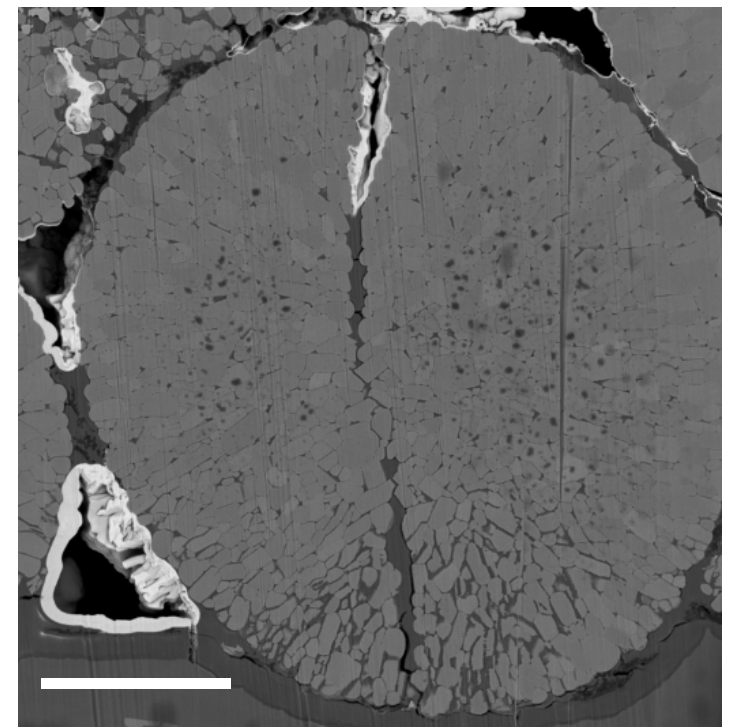

Figure 5: High resolution image of crack network in single NMC particle. Scale bar is $5 \mu \mathrm{m}$.

We also observe that there is a layer of CBD between the NMC particles and the current collector. These regions tend to have a stronger $\mathrm{F}$ signal than a $\mathrm{C}$ signal (Fig. 3), indicating that they may be deficient in the conductive additive in the CBD phase (i.e. carbon black) and have a higher concentration of the polymer binder (i.e. PVDF). Such a binder distribution layer is likely formed during the electrode drying process and related to the processing parameters unique to any particular manufacturing approach. In this case, the BSE and EDS data will provide useful information for those focused on electrode manufacturing processes, along with additional useful information for mesoscale modelers who may want to assign graded properties to the CBD phase based on the local C content. Alternatively, the higher $\mathrm{F}$ signal could be a result of residual $\mathrm{LiPF}_{6}$ salt remaining from the electrode drying, which will be discussed further in the context of the small cracks in the larger particles.

While this kintsugi technique has eliminated the pore back issue in identifying CBD vs. pore phases, there are still pore backs observed within the Pt phase (see the red ring in Fig. 4b). These Pt pore backs show up either as a darker version of the bright white Pt phase or a very dark black for the deepest holes. In the EDS image (Fig. 3) there are no signs of CBD or NMC particle within these new Pt pore backs, rather they return no strong EDS signal.

These pore backs exist simply because we did not deposit enough Pt to completely fill the pores. However, segmentation is still straightforward as the $\mathrm{Pt}$ pore backs are surrounded by a ring of $\mathrm{Pt}$, which makes them easy to classify using the TWS tool. The results in Figs. 4e to $4 \mathrm{~h}$ highlight the effectiveness of the approach. Although it was found to be unnecessary, the pores could be completely filled by either depositing thicker Pt layers or performing more deposition and milling cycles. This would also become easier if one were to create 3D images with FIB-SEM serial sectioning, since the additional deposition steps at each FIB slice would sequentially fill in the pores more. However, a recent paper by the Kench and Cooper suggests that capturing a large, high quality 2D image may be more valuable than a $3 \mathrm{D}$ volume with the equivalent number of pixels, as the 2D image will contain more information and can then be used to generate more representative 3D volumes [40]. Furthermore, Dahari et al. [41] have recently extended this work to fuse low and high resolution image together, providing an ideal use case for the Kintsugi method.

Some of the NMC particles contained features that are slightly darker than the surrounding NMC and only a few pixels across, as can be seen in Fig. 4b. As they are not white, it suggests that they are not empty cracks and although they could be connected to a percolating network filled with binder (requiring 3D data to validate), this initially seems unlikely so deep within a particle. Some of the observed contrast may simply be the result of grain boundaries interacting differently with the SEM beam than the adjacent grains. However, the higher resolution image in Fig. 5 suggests that at least the larger of these small features (several pixels across) towards the bottom of the particle are indeed filled with something. This seems unlikely to be binder as the electrodes are dried (and therefor the binder is in place and solid) before calendaring occurs, which was likely when these cracks were formed. One hypothesis as that these cracks are filled with $\mathrm{LiPF}_{6}$ salt that was not fully removed by the rinse step before drying these electrodes. This aligns with the EDS data which shows a strong $\mathrm{F}$ signal, but a weak $\mathrm{C}$ signal in this region. It is interesting to note that at this higher resolution, new dark features appear at the centre of individual grains, which are likely caused by charging due to the relatively poor electronic conductivity of the NMC.

Whatever the explanation for the smallest dark features, there will always be a degree of uncertainty when interpreting features on the order of 1 voxel across. In terms of the impact on metric extraction, it would be straightforward to remove features below a certain size when preparing the volume for use as a simulation domain, but we have left them visible in Fig. 4h for interest. It would of course be possible to extract a selection of conventional microstructural metrics from this data (such as porosity and interfacial area); however, these would only offer insight into a particular electrode (arbitrary in this case), rather than any additional information about the imaging technique, so we have chosen not to include them as it would only distract from the central message.

The approach presented in this paper can facilitate the interpretation of other surface imaging techniques, such as electron back-scatter diffraction (EBSD), which can offer information about elemental distribution and crystallographic orientation. Although the kintsugi method can greatly improve the contrast between phases, the authors would still strongly caution against the use of simple greyscale threshold based 
segmentation methods for an system with more than two phases. Even if the three phases appear to occupy distinct regions of the histogram, pixels at the interface between the brightest and darkest phases always run the risk of being wrongly attributed to the middle brightness phase. Furthermore, the convolution TWS segmentation method will essentially learn to use thresholding, if brightness is the best or only indicator of phase, so thresholding can be thought of as a very limited special case of the TWS.

\section{Conclusions}

In this paper, we demonstrated a novel approach to enhance the quality of FIB-SEM derived images of porous media. Through the in situ infiltration of platinum into the pores, the kintsugi method significantly reduces the ambiguity when differentiating between materials present on the imaging plane and those on the back wall of pores. This is of particular relevance to the characterisation of porous electrodes for lithiumion batteries. The imaging and segmentation of a cross section of a porous lithium-ion battery cathode is used to demonstrate the power of the technique. It is also compatible with, and could possibly be enhanced by, the standard FIB-SEM serial sectioning techniques for generating 3D volumes, although care would need to be taken to control slice thickness. This process could in principle be fully automated and this will be explored in a future study.

With this technique, we were able to identify intraparticle cracks and determine which were empty and which filled with CBD. In addition to enabling visual inspecting of the images, this technique, when combined with our machine learning-based multi-channel segmentation concept, enabled a high quality segmentation of the image into active material, CBD, pore, and current collector phases. The segmented data provides direct insight to qualitatively interpret the structure-performance of such electrodes. Furthermore, it can also feed into detailed image-based simulations to predict electrode performance.

\section{Data Availability}

All of the data associated with this paper can be found at https://doi.org/10.5281/zenodo.6815046.

\section{Acknowledgement}

This work was supported in part by funding from the EPSRC Faraday Institution Multi-Scale Modelling project (https://faraday.ac.uk/; EP/S003053/1, grant number FIRG003 received by SJC). This paper describes objective technical results and analysis. Any subjective views or opinions that might be expressed in the paper do not necessarily represent the views of the U.S. Department of Energy or the United States Government. SAR was supported by the Laboratory Di- rected Research and Development program at Sandia National Laboratories, a multimission laboratory managed and operated by National Technology and Engineering Solutions of Sandia, LLC., a wholly owned subsidiary of Honeywell International, Inc., for the U.S. Department of Energy's National Nuclear Security Administration under contract DE-NA-0003525.

\section{References}

[1] Mark E. Ferraro, Bradley L. Trembacki, Victor E. Brunini, David R. Noble, and Scott A. Roberts. Electrode mesoscale as a collection of particles: Coupled electrochemical and mechanical analysis of NMC cathodes. Journal of The Electrochemical Society, 167(1):013543, jan 2020. doi: 10.1149/1945-7111/ab632b.

[2] Tuan-Tu Nguyen, Arnaud Demortière, Benoit Fleutot, Bruno Delobel, Charles Delacourt, and Samuel J Cooper. The electrode tortuosity factor: why the conventional tortuosity factor is not well suited for quantifying transport in porous li-ion battery electrodes and what to use instead. $n p j$ Computational Materials, 6(1):1-12, 2020.

[3] Lukas Zielke, Tobias Hutzenlaub, Dean R. Wheeler, Chien-Wei Chao, Ingo Manke, André Hilger, Nils Paust, Roland Zengerle, and Simon Thiele. Three-phase multiscale modeling of a licoo2 cathode: Combining the advantages of fib-sem imaging and x-ray tomography. $A d$ vanced Energy Materials, 5(5):1401612, 2015. doi: 10.1002/aenm.201401612.

[4] Bradley L. Trembacki, Aashutosh N. Mistry, David R. Noble, Mark E. Ferraro, Partha P. Mukherjee, and Scott A. Roberts. Mesoscale analysis of conductive binder domain morphology in lithium-ion battery electrodes. Journal of The Electrochemical Society, 165(13):E725E736, 2018. doi: 10.1149/2.0981813jes.

[5] Martin Ebner, Felix Geldmacher, Federica Marone, Marco Stampanoni, and Vanessa Wood. X-ray tomography of porous, transition metal oxide based lithium ion battery electrodes. Advanced Energy Materials, 3(7):845850 , 2013. ISSN 1614-6840. doi: 10.1002/aenm. 201200932.

[6] Patrick Pietsch and Vanessa Wood. X-ray tomography for lithium ion battery research: A practical guide. Annual Review of Materials Research, 47(1):451-479, 2017. doi: 10.1146/ annurev-matsci-070616-123957.

[7] James R. Wilson, J. Scott Cronin, Scott A. Barnett, and Stephen J. Harris. Measurement of three-dimensional microstructure in a licoo2 positive electrode. Journal of Power Sources, 196: 3443 - 3447, 2011. doi: https://doi.org/10.1016/ j.jpowsour.2010.04.066.

[8] Zhao Liu, J. Scott Cronin, Yu chen K. ChenWiegart, James R. Wilson, Kyle J. YakalKremski, Jun Wang, Katherine T. Faber, and 
Scott A. Barnett. Three-dimensional morphological measurements of licoo 2 and licoo2/li(ni1/3mn1/3co1/3)o2 lithium-ion battery cathodes. Journal of Power Sources, 227:267 - 274, 2013. doi: https://doi.org/10.1016/j. jpowsour.2012.11.043.

[9] Barnaby D.A. Levin. Direct detectors and their applications in electron microscopy for materials science. Journal of Physics: Materials, 4(4): 042005, 2021.

[10] Efrat Shefer, Ami Altman, Rolf Behling, Raffy Goshen, Lev Gregorian, Roterman. Yalon, Igor Uman, Naor Wainer, Yoad Yagil, and Oren Zarchin. State of the art of ct detectors and sources: A literature review. Curr Radiol Rep, 1:76-91, 2013.

[11] Takao Marui, Ranjan K. Badheka, and Frank H. Read. Magnetic immersion lense with detection arrangement, dec 2003. US Patent US6,664,544 B1.

[12] YK. Chen-Wiegart, Zhao Liu, Faber T.K., Bernett SA., and Wang J. 3d analysis of a licoo2-li(ni1/3mn1/3co1/3)o2 li-ion battery positive electrode using $\mathrm{x}$-ray nano-tomography. Electrochemistry Communications, 28, 2013. doi: http://dx.doi.org/10.1016/j.elecom.2012.12. 021.

[13] Zhisen Jiang, Jizhou Li, Yang Yang, Linqin $\mathrm{Mu}$, Chenxi Wei, Xiqian Yu, Piero Pianetta, Kejie Zhao, Peter Cloetens, Feng Lin, and Yijin Liu. Machine-learning-revealed statistics of the particle-carbon/binder detachment in lithium-ion battery cathodes. Nature Communications, 11 (1):2310, May 2020. ISSN 2041-1723. doi: 10.1038/s41467-020-16233-5.

[14] Tomas Radlicka, Marek Uncovsky, and Martin Oral. In lens bse detector with energy filtering. Ultramicroscopy, 189:102-108, 2018.

[15] TL Burnett and PJ Withers. Completing the picture through correlative characterization. Nature materials, 18(10):1041-1049, 2019.

[16] Tuan-Tu Nguyen, Julie Villanova, Zeliang Su, Rémi Tucoulou, Benoît Fleutot, Bruno Delobel, Charles Delacourt, and Arnaud Demortière. 3D Quantification of Microstructural Properties of LiNi 0.5 Mn 0.3 Co 0.2O2 High-Energy Density Electrodes by X-Ray Holographic NanoTomography. Advanced Energy Materials, page 2003529, jan 2021. ISSN 1614-6832. doi: 10.1002/aenm.202003529.

[17] P. Pietsch, M. Ebner, F. Marone, M. Stampanoni, and V. Wood. Determining the uncertainty in microstructural parameters extracted from tomographic data. Sustainable Energy Fuels, 2:598605, 2018. doi: 10.1039/C7SE00498B.

[18] Michael C. Krygier, Tyler LaBonte, Carianne Martinez, Chance Norris, Krish Sharma, Lincoln N. Collins, Partha P. Mukherjee, and Scott A. Roberts. Quantifying the unknown impact of segmentation uncertainty on image-based simulations. Nature Communications, 12, 2021. doi: 10.1038/s41467-021-25493-8.

[19] Samantha L. Morelly, Jeff Gelb, Francesco Iacoviello, Paul R. Shearing, Stephen J. Harris, Nicolas J. Alvarez, and Maureen H. Tang. Threedimensional visualization of conductive domains in battery electrodes with contrast-enhancing nanoparticles. ACS Applied Energy Materials, 1 (9):4479-4484, sep 2018. doi: 10.1021/acsaem. $8 \mathrm{~b} 01184$.

[20] Bradley L. Trembacki, David R. Noble, Victor E. Brunini, Mark E. Ferraro, and Scott A. Roberts. Mesoscale effective property simulations incorporating conductive binder. Journal of The Electrochemical Society, 164(11):E3613E3626, 2017. doi: 10.1149/2.0601711jes.

[21] Aashutosh N. Mistry, Kandler Smith, and Partha P. Mukherjee. Secondary-phase stochastics in lithium-ion battery electrodes. ACS Applied Materials \& Interfaces, 10(7):6317-6326, 2018. doi: 10.1021/acsami.7b17771.

[22] Ken Mingard, Helen G. Jones, and Mark Gee. Metrological challenges for reconstruction of 3$\mathrm{d}$ microstructures by focused ion beam tomography methods. Journal of Microscopy, 2, 93 - 108, 2014.

[23] Zhao Liu, YK. Chen-Wiegart, Wang J., Bernett SA., and Faber T.K. Three-phase 3d reconstruction of a licoo2 cathode via fib-sem tomography. Microscopy and Microanalysis, 22, 2016. doi: https://doi.org/10.1017/S1431927615015640.

[24] Tim Burnett, Ron Kelley, Winiarski B., Contreras L., Daly M., Gholinia A., Burke MG., and Withers PJ. Large volume serial section tomography by xe plasma fib dual beam microscopy. Ultramicroscopy, 161:119 - 129, 2016. doi: 10.1016/j.ultramic.2015.11.001.

[25] Bartłomiej Winiarski and Remco Geurts. Laser xe+ plasma fib-sem: Correlative microscopy of $3 \mathrm{~d}$ microstructures from nanometers to millimeters. Microscopy and Analysis, EMEA:S3-S8, 2020.

[26] Masashi Kishimoto, Marina Lomberg, Enrique Ruiz-Trejo, and Nigel P. Brandon. Enhanced triple-phase boundary density in infiltrated electrodes for solid oxide fuel cells demonstrated by high-resolution tomography. Journal of Power Sources, 266:291-295, 2014. doi: 10.1016/j. jpowsour.2014.05.038.

[27] Sourov Ghosh, Hidenori Ohashi, Hiroshi Tabata, Yoshiyuki Hashimasa, and Takeo Yamaguchi. Microstructural pore analysis of the catalyst layer in a polymer electrolyte membrane fuel cell: A combination of resin pore-filling and FIB/SEM. International Journal of Hydrogen Energy, 40 (45):15663-15671, dec 2015. ISSN 03603199. doi: 10.1016/j.ijhydene.2015.09.080.

[28] Moshiel Biton, Vladimir Yufit, Farid Tariq, Masashi Kishimoto, and Nigel Brandon. Enhanced Imaging of Lithium Ion Battery Electrode Materials. Journal of The Electrochemical Society, 164(1):A6032-A6038, 2017. ISSN 0013- 
4651. doi: 10.1149/2.0061701jes.

[29] Moses Ender, Jochen Joos, Thomas Carraro, and Ellen Ivers-Tiffée. Quantitative characterization of lifepo 4 cathodes reconstructed by fib/sem tomography. Journal of The Electrochemical Society, 159:A972, 2012. doi: https://doi.org/10. 1149/2.033207jes.

[30] Marie Francine Lagadec, Martin Ebner, Raphael Zahn, and Vanessa Wood. Communication-technique for visualization and quantification of lithium-ion battery separator microstructure. Journal of The Electrochemical Society, 163(6):A992-A994, 2016. doi: 10.1149/ 2.0811606jes. URL https://doi.org/10. $1149 / 2.0811606 j$ jes.

[31] Marie Francine Lagadec, Raphael Zahn, and Vanessa Wood. Characterization and performance evaluation of lithium-ion battery separators, jan 2019. ISSN 20587546.

[32] Severin Vierrath, Lukas Zielke, Riko Moroni, Andrew Mondon, Dean R. Wheeler, Roland Zengerle, and Simon Thiele. Morphology of nanoporous carbon-binder domains in Li-ion batteries-A FIB-SEM study. Electrochemistry Communications, 60:176-179, nov 2015. ISSN 1388-2481. doi: 10.1016/J.ELECOM.2015.09. 010.

[33] Riko Moroni and Simon Thiele. FIB/SEM tomography segmentation by optical flow estimation. Ultramicroscopy, 219:113090, dec 2020. ISSN 18792723. doi: 10.1016/j.ultramic.2020. 113090.

[34] Ignacio Arganda-Carreras, Verena Kaynig, Curtis Rueden, Kevin W Eliceiri, Johannes Schindelin, Albert Cardona, and $\mathrm{H}$ Sebastian Seung. Trainable weka segmentation: a machine learning tool for microscopy pixel classification. Bioinformatics, 33(15):2424-2426, 2017.

[35] Simon Müller, Christina Sauter, Ramesh Shunmugasundaram, Nils Wenzler, Vincent De Andrade, Francesco De Carlo, Ender Konukoglu, and Vanessa Wood. Deep learning-based seg- mentation of lithium-ion battery microstructures enhanced by artificially generated electrodes. Nature Communications 2021 12:1, 12(1):1-12, oct 2021. ISSN 2041-1723. doi: 10.1038/ s41467-021-26480-9.

[36] Santhana K. Eswara-Moorthy, Prasanth Balasubramanian, Willem Van Mierlo, Jörg Bernhard, Mario Marinaro, Margret Wohlfahrt-Mehrens, Ludwig Jörissen, and Ute Kaiser. An In Situ SEM-FIB-Based Method for Contrast Enhancement and Tomographic Reconstruction for Structural Quantification of Porous Carbon Electrodes. Microscopy and Microanalysis, 20(5): 1576-1580, apr 2014. ISSN 1431-9276. doi: 10.1017/S1431927614012884.

[37] B. Winiarski, G. Pyka, C.A. Wade, A. Chirazi, D. Lichau, G.M. Burke, and P.J. Withers. Investigation of cracking in additively manufactured in718 by correlative tomography. Proceedings of Microscopy and Microanalysis, 24: 366 - 367, 2018. doi: https://doi.org/10.1017/ S1431927618002325.

[38] Johannes Schindelin, Ignacio Arganda-Carreras, Erwin Frise, Verena Kaynig, Mark Longair, Tobias Pietzsch, Stephan Preibisch, Curtis Rueden, Stephan Saalfeld, and Benjamin Schmid. Fiji: an open-source platform for biological-image analysis. Nature methods, 9(7):676-682, 2012.

[39] Michael D Abràmoff, Paulo J Magalhães, and Sunanda J Ram. Image processing with imagej. Biophotonics international, 11(7):36-42, 2004.

[40] Steve Kench and Samuel J. Cooper. Generating three-dimensional structures from a twodimensional slice with generative adversarial network-based dimensionality expansion. Nature Machine Intelligence, 3(4):1-7, feb 2021. ISSN 2522-5839.

[41] Amir Dahari, Steve Kench, Isaac Squires, and Samuel J Cooper. Super-resolution of multiphase materials by combining complementary $2 \mathrm{~d}$ and $3 \mathrm{~d}$ image data using generative adversarial networks. arXiv preprint arXiv:2110.11281, 2021. 